\title{
Safe Distal Resection Margin in Patients With T3 Mid and Distal Rectal Cancer Who Underwent a Sphincter-Saving Resection Without Preoperative Radiotherapy
}

\author{
Bong Hwa Lee, Hyoung Chul Park, Min Jeong Kin, Mi Young Jang \\ Department of Surgery, Hallym University Sacred Heart Hospital, Hallym University College of Medicine, Anyang, Korea
}

\section{See Article on Page 231-237}

The Korean Clinical Practice Guideline for Colon and Rectal Cancer v.1.0 (2012) recommends preoperative chemoradiation as an initial treatment for patients with clinical stage II and III (cT3+ or $\mathrm{cN}+$ ) rectal cancer [1]. The National Comprehensive Cancer Network guideline v.4. (2013) for rectal cancer also recommends preoperative chemoradiation as an initial treatment for T3N0 or

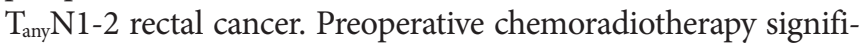
cantly decreases the local recurrence rate after a total mesorectal excision (TME) curative resection for rectal cancer, but the functional disability of the anal sphincter after radiation therapy cannot be neglected. In Japan, a major trend in the treatment of rectal cancer has been the radical low anterior resection including lateral node dissection without adjuvant radiotherapy.

The Study Group of the Japanese Society for Cancer of the Colon and Rectum reported on the Clinical Significance of the Mesorectal Extension of Rectal Cancer that the distance of mesorectal extension (DME), which was measured with histology after fixation in $20 \%$ formalin, was a significant prognostic factor for recurrence-free survival (cut off point, $4 \mathrm{~mm}$ ) [2]. The multivariate Cox regression analysis showed that the recurrence-free fiveyear survival rate was significantly higher in patients with a DME $\leq 4 \mathrm{~mm}$ than in patients with DME $>4 \mathrm{~mm}$ in stage IIA and IIIA cancer, but not in stage IIIC cancer $(\mathrm{P}=0.00015, \mathrm{P}=0.0001$, and $\mathrm{P}=0.2697$, respectively). The reports suggested that local recurrences were increased by the status of cancer-positive lymph

Correspondence to: Bong Hwa Lee, M.D.

Department of Surgery, Hallym University Hospital, Hallym University

College of Medicine, 22 Gwanpyeong-ro 170beon-gil, Dongan-gu,

Anyang 431-796, Korea

Tel: +82-31-380-3772, Fax: +82-31-380-4118

E-mail: bshlee@hallym.ac.kr

(C) 2013 The Korean Society of Coloproctology

This is an open-access article distributed under the terms of the Creative Commons Attribution NonCommercial License (http://creativecommons.org/licenses/by-nc/3.0) which permits unrestricted noncommercial use, distribution, and reproduction in any medium, provided the original work is properly cited. nodes even in the same category of T3 cancer in patients without radiation therapy.

The distal resection margin (DRM) means the length of the tumor-free normal mucosa and rectal wall. Han et al. [3] published the manuscript, 'Association between a close distal resection margin and recurrence after sphincter-saving resections for T3 midor low-rectal cancer without radiotherapy' With retrospective data, they described the 5-year local recurrence rates as $6.69 \%$ in patient with $\mathrm{DRM} \leq 10 \mathrm{~mm}$ and $9.52 \%$ in patients with $\mathrm{DRM}>10$ $\mathrm{mm}$, respectively (DRM cut off point, $1 \mathrm{~cm}$; $\mathrm{P}=0.3981$ ). However, they gave no descriptions of cancer-positive lymph nodes in the groups with stage IIA, IIIB, and IIIC cancer. The local recurrence may be influenced not only by DRM but also by the status of lymph nodes. A Norwegian national study recommended a DRM of $>10 \mathrm{~mm}$ for rectal cancer patients treated without radiotherapy [4]. The 5-year local recurrence rate was $14.5 \%$ for patients with DRM of $0-10 \mathrm{~mm}$ compared to $8.66 \%$ for patients with DRM $>11 \mathrm{~mm}(\mathrm{P}<0.001)$. The proportions of T3 cancer were $48 \%$ and $63 \%$, respectively, in those two groups. Current Japanese guidelines state a $2-\mathrm{cm}$ DRM is needed for rectal cancer with a distal edge below the peritoneal reflection. Intramural and extramural distal spread prevents local control and promotes systemic metastasis. If the specimen is not pinned, the length of the DRM can shrink by $50 \%$ in a nonradiated rectum after formalin fixation. A gross determination of a safe distal resection margin is difficult in the operating room. The frozen-section result for the DRM may be falsely negative in $12 \%$ of all cases.

A systemic review supported the practice of sphincter preservation in selected settings of close distal resection margins $(\leq 1 \mathrm{~cm})$ after mesorectal excision for distal rectal cancer [5]. A further subgroup study suggested that margins as close as $\leq 5 \mathrm{~mm}$, if they were indeed negative, might be acceptable. However, the importance of patient and tumor selection for this approach must be emphasized.

In distal rectal cancer, the mesorectal fascia does not extend beyond the puborectalis level, and the anal sphincter is another barrier for safe distal resection margins. Local recurrence may de- 


\section{Coloproctology Bong Hwa Lee, et al.}

velop with uninvolved margins related to lymphatic spread from the DRM. In preoperative radiation cases, a distal clearance margin of $1 \mathrm{~cm}$ or less may a safe distance in patients who undergo lower rectum cancer surgery [6].

We would like to conclude with the following remark. Although reduction of the DRM to less than $10 \mathrm{~mm}$ does not increase local recurrences in many low-risk patients, a DRM $>10 \mathrm{~mm}$ is safe in cases of rectal cancer with lymphovascular invasion, perineural invasion and multiple lymph-node metastases.

\section{REFERENCES}

1. Korean Academy of Medical Science. Korean clinical practice guideline for colon and rectal cancer v.1.0. Seoul: Korean Academy of Medical Science; 2012.

2. Akagi Y, Shirouzu K, Fujita S, Ueno H, Takii Y, Komori K, et al. Predicting oncologic outcomes by stratifying mesorectal exten- sion in patients with pT3 rectal cancer: a Japanese multi-institutional study. Int J Cancer 2012;131:1220-7.

3. Han JW, Lee MJ, Park HK, Shin JH, An MS, Ha TK, et al. Association between a close distal resection margin and recurrence after sphincter-saving resections for T3 mid- or low-rectal cancer without radiotherapy. Ann Coloproctol 2013;29:231-7.

4. Bernstein TE, Endreseth BH, Romundstad P, Wibe A; Norwegian Colorectal Cancer Registry. What is a safe distal resection margin in rectal cancer patients treated by low anterior resection without preoperative radiotherapy? Colorectal Dis 2012;14:e48-55.

5. Bujko K, Rutkowski A, Chang GJ, Michalski W, Chmielik E, Kusnierz J. Is the $1-\mathrm{cm}$ rule of distal bowel resection margin in rectal cancer based on clinical evidence? A systematic review. Ann Surg Oncol 2012;19:801-8.

6. Penninckx F. The safe distal tumour-free margin after sphincter preserving resection for rectal cancer: an ongoing debate. Colorectal Dis 2012;14:131-2. 